\title{
The disabilities of the arm, shoulder and hand (DASH) outcome questionnaire: longitudinal construct validity and measuring self-rated health change after surgery
} Christina Gummesson*1,2, Isam Atroshi ${ }^{2}$ and Charlotte Ekdahl ${ }^{1}$

\author{
Address: ${ }^{1}$ Department of Physical Therapy, Lund University, Lund, Sweden and ${ }^{2}$ Department of Orthopedics, Hässleholm-Kristianstad Hospitals, \\ Kristianstad, Sweden \\ Email: Christina Gummesson* - christina.gummesson@sjukgym.lu.se; Isam Atroshi - isam.atroshi@mailbox.swipnet.se; \\ Charlotte Ekdahl - charlotte.ekdahl@sjukgym.lu.se \\ * Corresponding author
}

Published: 16 June 2003

BMC Musculoskeletal Disorders 2003, 4: I I
Received: 09 January 2003

Accepted: 16 June 2003

This article is available from: http://www.biomedcentral.com/I47/-2474/4/I I

(C) 2003 Gummesson et al; licensee BioMed Central Ltd. This is an Open Access article: verbatim copying and redistribution of this article are permitted in all media for any purpose, provided this notice is preserved along with the article's original URL.

\begin{abstract}
Background: The disabilities of the arm, shoulder and hand (DASH) questionnaire is a selfadministered region-specific outcome instrument developed as a measure of self-rated upperextremity disability and symptoms. The DASH consists mainly of a 30 -item disability/symptom scale, scored 0 (no disability) to 100. The main purpose of this study was to assess the longitudinal construct validity of the DASH among patients undergoing surgery. The second purpose was to quantify self-rated treatment effectiveness after surgery.
\end{abstract}

Methods: The longitudinal construct validity of the DASH was evaluated in 109 patients having surgical treatment for a variety of upper-extremity conditions, by assessing preoperative-topostoperative (6-2I months) change in DASH score and calculating the effect size and standardized response mean. The magnitude of score change was also analyzed in relation to patients' responses to an item regarding self-perceived change in the status of the arm after surgery. Performance of the DASH as a measure of treatment effectiveness was assessed after surgery for subacromial impingement and carpal tunnel syndrome by calculating the effect size and standardized response mean.

Results: Among the 109 patients, the mean (SD) DASH score preoperatively was 35 (22) and postoperatively 24 (23) and the mean score change was 15 (13). The effect size was 0.7 and the standardized response mean I.2.

The mean change ( $95 \%$ confidence interval) in DASH score for the patients reporting the status of the arm as "much better" or "much worse" after surgery was 19 (15-23) and for those reporting it as "somewhat better" or "somewhat worse" was $10(7-14)(p=0.01)$. In measuring effectiveness of arthroscopic acromioplasty the effect size was 0.9 and standardized response mean 0.5 ; for carpal tunnel surgery the effect size was 0.7 and standardized response mean 1.0.

Conclusion: The DASH can detect and differentiate small and large changes of disability over time after surgery in patients with upper-extremity musculoskeletal disorders. A 10-point difference in mean DASH score may be considered as a minimal important change. The DASH can show treatment effectiveness after surgery for subacromial impingement and carpal tunnel syndrome. The effect size and standardized response mean may yield substantially differing results. 


\section{Background}

The disability of the arm, shoulder and hand (DASH) questionnaire is an upper-extremity specific outcome measure that was introduced by the American Academy of Orthopedic Surgeons in collaboration with a number of other organizations[1]. The rationale behind the use of one outcome measure for different upper extremity disorders is that the upper extremity is a functional unit[2]. In this respect, the DASH would be suitable because of its property of being mainly a measure of disability. In addition to decreasing the administrative burden associated with using different disease-specific measures, one of the main concepts behind developing the DASH was to facilitate comparisons among different upper-extremity conditions in terms of health burden[1]. The DASH is now available in several languages http://www.dash.iwh.on.ca, and studies of reliability and validity have been published for the original version[3] as well as for the German[4], Italian[5], Spanish[6] and Swedish[7] versions. In addition, research studies regarding a French[8] and a Dutch[9] version of the DASH have been published.

The DASH is being increasingly used in cross-sectional studies. To enhance the use of the DASH in prospective studies (such as assessment of effectiveness of different treatment methods) further studies of the instrument's ability to detect change over time would be helpful both for interpretation of score changes and for sample size calculations. Different aspects of an instrument's ability to measure change have been highlighted including studying changes over time for groups or individuals and comparing groups at one occasion[10]. The analysis of score change is commonly referred to as responsiveness[11$15]$, but the term longitudinal construct validity has also been used[16] and it has been advocated that responsiveness is a part of the validity analysis[15]. There is no consensus on the nomenclature or the appropriate statistical analysis and different suggestions have been made $[12,17-19]$. To facilitate prospective research, longitudinal studies of the instrument's ability to detect changes and identify smaller and larger changes in health status as perceived by the patient are needed.

We believe that the concept of detecting change over time is part of the validity assessment and therefor may be referred to as longitudinal construct validity. To date, we have found only one published study concerning the longitudinal construct validity of the DASH in a variety of orthopedic disorders of the upper extremity[3]. Considering the nature of the instrument, longitudinal construct validity can be assessed among a group of patients with different upper extremity disorders. In contrast, when using the instrument in patients with a particular diagnosis the effectiveness of a specific treatment can be assessed. To analyze treatment effectiveness the direction of change becomes important, as opposed to analyzing longitudinal construct validity, which concerns the ability to detect change irrespective of whether the change is improvement or worsening. Therefor it would be important to study the longitudinal construct validity of the DASH as well as its performance as a measure of treatment effectiveness.

The main purpose of this study was to assess the longitudinal construct validity of the DASH among patients undergoing surgery for a variety of upper extremity disorders. The second purpose was to quantify self-rated treatment effectiveness after surgery for subacromial impingement and carpal tunnel syndrome when using the DASH. To ensure reliability of the DASH in this study we also aimed to determine the internal consistency of the scale in each patient population studied.

\section{Methods \\ The DASH questionnaire}

The main part of the DASH is a 30-item disability/symptom scale concerning the patient's health status during the preceding week[20]. The items ask about the degree of difficulty in performing different physical activities because of the arm, shoulder, or hand problem (21 items), the severity of each of the symptoms of pain, activity-related pain, tingling, weakness and stiffness ( 5 items), as well as the problem's impact on social activities, work, sleep, and self-image (4 items). Each item has five response options. The scores for all items are then used to calculate a scale score ranging from 0 (no disability) to 100 (most severe disability). The score for the disability/symptom scale is called the DASH score. In this study we used the Swedish version of the DASH[7].

\section{Patients}

Patients with upper-extremity musculoskeletal conditions planned for surgical treatment at an orthopedic department were considered for inclusion in this study. Exclusion criteria were age below 18 years, symptom duration of less than 2 months, or inability to complete questionnaires due to cognitive impairment or language difficulties. The DASH was completed preoperatively by 118 consecutive eligible patients[7]. Postoperatively, 9 (8\%) of the patients did not respond and the remaining 109 patients completed the DASH after a minimum followup time of 6 months (Table 1). The 2 largest diagnostic groups comprised patients who had undergone arthroscopic acromioplasty because of subacromial impingement and open carpal tunnel release because of carpal tunnel syndrome. Complete followup could be obtained for all patients in these 2 subgroups (Table 2).

The followup questionnaire also included an item regarding change in health status after surgery. It inquired about the status of the operated arm compared to its status 
Table I: Characteristics of all the responders and the two largest subgroups who completed the DASH before and after surgery, and the patients who did not respond after surgery (dropouts)

\begin{tabular}{lcccc}
\hline & $\begin{array}{c}\text { All responders } \\
\mathrm{n}=109\end{array}$ & $\begin{array}{c}\text { Arthroscopic acromioplasty } \\
\mathrm{n}=25\end{array}$ & $\begin{array}{c}\text { Carpal tunnel release } \\
\mathrm{n}=19\end{array}$ & $\begin{array}{c}\text { Dropouts } \\
\mathrm{n}=9\end{array}$ \\
\hline Women $(\mathrm{n})$ & 63 & 12 & 17 & 3 \\
Men $(\mathrm{n})$ & 46 & 13 & 2 & 6 \\
Age: mean (range) years & $52(18-83)$ & $54(37-71)$ & $52(30-83)$ & $56(24-76)$ \\
Followup: mean (range) months & $12(6-21)$ & $13(9-21)$ & $9(6-11)$ & - \\
\hline
\end{tabular}

Table 2: Patient diagnoses

\begin{tabular}{lcc}
\hline Diagnostic Group & Responders (n) & Dropouts (n) \\
\hline Subacromial impingement & 25 & 1 \\
Other shoulder disorders & 3 & 1 \\
Tennis elbow & 3 & 3 \\
Cubital tunnel syndrome & 3 & 1 \\
Finger/Hand tumor & 4 & 1 \\
Wrist/hand ganglion & 7 & 2 \\
Carpal tunnel syndrome & 19 & 1 \\
Dupuytren's disease & 13 & \\
Tenosynovitis & 10 & 2 \\
Sequelae tendon laceration & 4 & 6 \\
Trapeziometacarpal arthritis & 6 & \\
Other & 12 & \\
\hline
\end{tabular}

preoperatively (5 response options: much better, somewhat better, unchanged, somewhat worse, much worse). This item was accidentally missing in the initially mailed questionnaires and was therefore only completed by the last 83 participants.

\section{Analyses}

To assess one aspect of the reliability of the DASH scale when used in this patient population, the internal consistency was calculated using Cronbach alpha[21] for the total population as well as for the subgroups with subacromial impingement and carpal tunnel syndrome. For each of these populations, preoperative, postoperative and change scores were computed for the DASH. These scores were subjected to the one-sample KolmogorovSmirnov test to assess normality of distribution. As a measure of longitudinal construct validity, the effect size and standardized response mean were calculated for the DASH disability/symptom scale. The effect size was calculated as the mean difference between the baseline scores and the followup scores (i.e., mean change scores) divided by the standard deviation of the baseline scores. The standardized response mean was calculated as the mean change scores divided by the standard deviation of the change scores.
As external criterion for change in health status after surgery the item regarding how the patient rated the status of the operated arm compared to its status preoperatively was used. Because detecting both improvement and worsening reflect longitudinal construct validity, the preoperative-to-postoperative score differences were considered to be in the same direction and the mean change in DASH score and the 95\% confidence interval (CI) was calculated for the patients with the responses of "much better" or "much worse" and those with the responses "somewhat better" or "somewhat worse". The difference in the mean change scores between these two groups was assessed with the t-test.

For patients who reported that no change had occurred, the mean change in DASH score and the $95 \%$ CI were calculated (scores used in their actual direction).

The mean change in DASH score for the patients who did not and those who did receive the transition item regarding change in the status of the operated arm was compared with the t-test.

To assess the size of health change after surgery for subacromial impingement and carpal tunnel syndrome (i.e., 
Table 3: Internal consistency of the disabilities of the arm, shoulder and hand (DASH) questionnaire measured with the Cronbach alpha coefficient

\begin{tabular}{lcc}
\hline Population & DASH disability/symptoms scale & Postoperative \\
\cline { 2 - 3 } & Preoperative & 0.98 \\
\hline Total population $(n=109)$ & 0.97 & 0.97 \\
Arthroscopic acromioplasty $(\mathrm{n}=25)$ & 0.92 & 0.98 \\
Carpal tunnel release $(\mathrm{n}=19)$ & 0.96 & \\
\hline
\end{tabular}

Table 4: Results of the disabilities of the arm, shoulder and hand (DASH) disability/symptoms scale shown as scores, effect size (ES) and standardized response mean (SRM)

\begin{tabular}{|c|c|c|c|c|c|c|c|c|}
\hline \multirow{2}{*}{ Population } & \multicolumn{2}{|c|}{ Preoperative* } & \multicolumn{2}{|c|}{ Postoperative* } & \multicolumn{2}{|c|}{ Change } & \multirow{2}{*}{ ES } & \multirow[t]{2}{*}{ SRM } \\
\hline & mean $(S D)$ & median & mean (SD) & median & mean (SD) & median & & \\
\hline \multirow[t]{2}{*}{ Total population $(n=109)$} & $35(22)$ & 36 & $24(23)$ & 16 & II (I7) & 8 & 0.5 & 0.6 \\
\hline & & & & & $15(13)^{\dagger}$ & $12 \dagger$ & 0.7 & 1.2 \\
\hline Arthroscopic acromioplasty $(n=25)$ & $43(13)$ & 45 & $32(25)$ & 24 & II (23) & 6 & 0.9 & 0.5 \\
\hline Carpal tunnel release $(n=19)$ & $41(20)$ & 40 & $28(26)$ & 15 & $13(14)$ & 10 & 0.7 & 1.0 \\
\hline Dropouts $(n=9)$ & $24(18)$ & 28 & & & & & & \\
\hline
\end{tabular}

*Higher score $(0-100)$ indicates greater disability †All changes in scores (improvement or worsening) calculated as having the same direction (to assess longitudinal validity of the DASH as opposed to assessing treatment effectiveness)

treatment effectiveness), the change scores were used in their actual direction and the effect size and standardized response mean were calculated.

The relationship between the DASH change score and time since surgery (months) was analyzed with the Pearson correlation coefficient (r).

\section{Results}

\section{Reliability}

The Cronbach alpha coefficient was above 0.9 for the DASH disability/symptoms scale indicating good internal consistency when used in this patient population (Table $3)$.

\section{Longitudinal construct validity}

Among the 109 participants the mean (SD) change in DASH score was 15 (13) when all changes in scores (improvement or worsening) were calculated as having the same direction. The effect size was 0.7 and standardized response mean 1.2 (Table 4).

Of the 83 patients who answered the transition item concerning self-rated change in disability and symptoms after surgery the mean change ( $95 \% \mathrm{CI}$ ) in DASH score in the 53 patients responding "much better" or "much worse" was 19 (15-23) and in the 21 patients responding "somewhat better" or "somewhat worse" was 10 (7-14), ( $\mathrm{p}=$ $0.01)$. For the 9 patients reporting no change in the status of the arm after surgery (clinically stable group) the mean change (95\% CI) in DASH score was -0.3 (-3.6-3.0).

The mean change $(95 \% \mathrm{CI})$ in DASH score for the patients who did not receive the transition item was 16 (11-20) and it was $15(12-18)$ for the patients who responded to the item $(\mathrm{p}=0.9)$.

\section{Comparison of measures of treatment effectiveness}

For the group with subacromial impingement treated with arthroscopic acromioplasty, the effect size was 0.9 and the standardized response mean 0.5 (Table 4 ). For the group with carpal tunnel syndrome treated with open carpal tunnel release, the effect size was 0.7 and the standardized response mean 1.0.

Correlation between score change and time since surgery Among all 109 patients, no correlation was found between the DASH change score and time since surgery ( $\mathrm{r}$ $=0.06, \mathrm{p}=0.56)$. The correlation was weak-to-moderate but statistically non-significant among the patients treated with arthroscopic acromioplasty $(\mathrm{r}=0.29, \mathrm{p}=0.15)$ and those treated with carpal tunnel release $(\mathrm{r}=0.34, \mathrm{p}=$ $0.16)$.

\section{Discussion}

The importance of monitoring the effectiveness of treatment is well recognized and furthermore is the foundation of evidence-based health care. For this purpose 
instruments that have the ability to detect changes and can differentiate a small difference from a large difference are needed. In a previous study, the DASH score change was reported for 172 patients with different upper extremity disorders (such as shoulder arthritis and carpal tunnel syndrome). The mean change between baseline and followup scores 12 weeks after treatment was 13 (SD 17), the effect size was 0.6 and the standardized response mean was $0.8[3]$. The changes were also shown for patients rating their problem as better (mean score change 17, effect size 0.75 , standardized response mean 1.1 ) and patients rating their function as better (mean score change 20, effect size 0.8, standardized response mean 1.2). Also, based on the results of the present study, it appears that the DASH has the ability to detect changes on group level corresponding to the patients' perception after surgery in a variety of upper extremity disorders. A significant difference in DASH scores between patients responding "much better/worse" and "somewhat better/worse" was found showing the instruments ability to discriminate between these degrees of change. A mean score change of 19 indicated a change in disability rated as "much better/worse" and a mean score change of 10 as "somewhat better/ worse". It has been suggested that the score change rated as "somewhat changed" could be defined as the limit for minimal important change[18]. This information could then be used for power calculations when planning prospective studies. In a recent study a DASH score change of 15 has been suggested to discriminate between improved and unimproved patients[3]. This was based on the patients' responses to a question about "being able to cope with the problem and do what you would like to do", with a response change from "not being able to cope" before treatment to "being able to cope" at followup considered as criterion for improvement[3]. However, we believe that a change in disability can be important even if the patients are not able to do all what they want to do or, at a particular time, not being able to cope with the problem. Future investigations are needed to determine whether the DASH is sensitive to milder degrees of impact other than that of surgery.

The difference noted in the group stating no change (mean score change -0.3) can be seen as the difference that occurred by chance and was similar to the score change previously reported[3,7]. A difference of this size should not be considered as a real change of upper extremity disability.

In the analysis of health transition only the last 83 patients were included because the item was accidentally missing in the initially mailed questionnaires. The mean change in DASH score did not significantly differ between the patients who did not receive and those who responded to the transition item suggesting that it is unlikely the missing item could have substantially influenced the results.

We chose to use self-rated change of health status in the operated arm as external criterion in order to ensure that it did not capture global health changes not related to the upper extremities.

The minimum followup time in the present study was 6 months and the latest response was received 21 months after surgery. The minimum followup time was chosen as it was expected to be sufficient to show improvement after surgery in most disorders. As shown in the correlation analysis time since surgery had, within this followup period, only weak-to-moderate but statistically non-significant association with the change in DASH scores after arthroscopic acromioplasty and carpal tunnel release. However, the difference in followup time is a limitation that can have implication, particularly when interpreting the size of change in DASH score for the assessment of treatment effectiveness. The possible implication of response shift also needs to be evaluated in future studies.

In this study the DASH demonstrated high Cronbach alpha values, indicating an excellent internal consistency that is adequate for group as well as for individual comparisons[22]. These results support the use of the DASH to measure changes in upper extremity function also on an individual level. However, for individual patient assessment with the DASH the magnitude of score change has to be studied on individual level[17]. It is important to note that in the present study only longitudinal construct validity on group level has been analyzed.

The treatment effectiveness calculations showed that for arthroscopic acromioplasty the effect size was larger than the standardized response mean, while for carpal tunnel release the opposite was shown. This illustrates the difficulties with interpretation of such calculations when only one of the analyses is presented. Since the effect size is dependent on the homogeneity of the group preoperatively and the standardized response mean is dependent on the homogeneity of the change of disability, these calculations will by nature differ in almost any group. Both calculation methods are common; however, little has been discussed about the limitations associated with these analyses, though it has been highlighted[15,18]. The use of the DASH in other populations of similar diagnostic groups and interventions is needed to show the degree of consistency in the estimates of treatment effectiveness.

\section{Conclusions}

The DASH can detect and differentiate small and large changes in disability over time after surgery in patients with upper extremity musculoskeletal disorders. A 10- 
point difference in mean DASH score might be considered as a minimal important change. The DASH can show selfrated treatment effectiveness after surgery for carpal tunnel syndrome and subacromial impingement. The effect size and standardized response mean (commonly used indices of the magnitude of health change measured by questionnaires) may yield substantially differing results.

\section{Competing interests}

None declared.

\section{Authors' contributions}

CG and IA participated in the design of the study, data collection and analysis, and writing of this manuscript. CE participated in the analysis and writing of this manuscript. All authors read and approved the final manuscript.

\section{Acknowledgments}

This study was supported by the Swedish Foundation for Health Care Sciences and Allergy Research (Vårdal Stiftelse), the Kristianstad County Council and the Skåne County Council.

We thank Gunilla B. Persson and Iréne Nordén for assistance with data collection, Ragnar Johnsson, MD, PhD, for his helpful comments, Gert-Uno Larsson, for his contribution, and Ewald Ornstein for his support. We acknowledge the work of the American Academy of Orthopedic Surgeons and the Institute for Work \& Health, Toronto, Canada, in developing the DASH.

\section{References}

I. Hudak PL, Amadio PC, Bombardier C and The Upper Extremity Collaborative Group (UECG): Development of an upper extremity outcome measure: the DASH (disabilities of the arm, shoulder and hand) [corrected] Am J Ind Med 1996, 29:602-608.

2. Davis AM, Beaton DE, Hudak P, Amadio P, Bombardier C, Cole D, Hawker G, Katz JN, Makela M, Marx RG, Punnett L and Wright JG: Measuring disability of the upper extremity: a rationale supporting the use of a regional outcome measure J Hand Ther 1999, I 2:269-274.

3. Beaton DE, Katz JN, Fossel AH, Wright JG, Tarasuk $V$ and Bombardier C: Measuring the whole or the parts? Validity, reliability, and responsiveness of the Disabilities of the Arm, Shoulder and Hand outcome measure in different regions of the upper extremity J Hand Ther 200I, I 4: I28-I 46.

4. Offenbaecher M, Ewert T, Sangha $O$ and Stucki G: Validation of a German version of the disabilities of arm, shoulder, and hand questionnaire (DASH-G) / Rheumatol 2002, 29:40 I-402.

5. Padua R, Padua L, Ceccarelli E, Romanini E, Zanoli G, Amadio PC and Campi A: Italian version of the disability of the arm, shoulder and hand (DASH) questionnaire. Cross-cultural adaptation and validation J Hand Surg Br 2003, 28: $179-186$.

6. Rosales RS, Delgado EB and De La Lastra-Bosch ID: Evaluation of the Spanish version of the DASH and carpal tunnel syndrome health-related quality-of-life instruments: Cross-cultural adaptation process and reliability J Hand Surg Am 2002, 27:334-343.

7. Atroshi I, Gummesson C, Andersson B, Dahlgren E and Johansson A: The disabilities of the arm, shoulder and hand (DASH) outcome questionnaire: reliability and validity of the Swedish version evaluated in 176 patients Acta Orthop Scand 2000, 7I:613-618.

8. Dubert $T$, Voche P, Dumontier $C$ and Dinh A: [The DASH questionnaire. French translation of a trans-cultural adaptation] Chir Main 200I, 20:294-302.

9. Veehof MM, Sleegers EJ, van Veldhoven NH, Schuurman $A H$ and van Meeteren NL: Psychometric qualities of the Dutch language version of the Disabilities of the Arm, Shoulder, and Hand questionnaire (DASH-DLV) J Hand Ther 2002, I5:347-354.

10. Beaton DE, Bombardier C, Katz JN, Wright JG, Wells G, Boers M, Strand $V$ and Shea B: Looking for important change/differences in studies of responsiveness. OMERACT MCID Working Group. Outcome Measures in Rheumatology. Minimal Clinically Important Difference J Rheumatol 200I, 28:400-405.

II. Deyo RA, Diehr P and Patrick DL: Reproducibility and responsiveness of health status measures: statistics and strategies for evaluation Control Clin Trials I99I, I2:142S-I58S.

12. Guyatt $G$, Walter $S$ and Norman $G$ : Measuring change over time: assessing the usefulness of evaluative instruments / Chronic Dis 1987, 40: $171-178$.

13. Ware JEJ, Kemp JP, Buchner DA, Singer AE, Nolop KB and Goss TF: The responsiveness of disease-specific and generic health measures to changes in the severity of asthma among adults Qual Life Res 1998, 7:235-244.

14. Wright JG and Young NL: A comparison of different indices of responsiveness J Clin Epidemiol 1997, 50:239-246.

15. Stratford PW, Binkley FM and Riddle DL: Health status measures: strategies and analytic methods for assessing change scores Phys Ther 1996, 76: I 109-1 I23.

16. Liang $\mathrm{MH}$ : Longitudinal construct validity: establishment of clinical meaning in patient evaluative instruments Med Care 2000, 38: $1184-1190$.

17. Beaton DE, Boers $M$ and Wells GA: Many faces of the minimal clinically important difference (MCID): a literature review and directions for future research Curr Opin Rheumatol 2002 14:109-114.

18. Husted JA, Cook RJ, Farewell VT and Gladman DD: Methods for assessing responsiveness: a critical review and recommendations J Clin Epidemiol 2000, 53:459-468.

19. Liang MH, Lew RA, Stucki G, Fortin PR and Daltroy L: Measuring clinically important changes with patient-oriented questionnaires Med Care 2002, 40:I145-II5I.

20. Solway S, Beaton DE, McConnell S and Bombardier C: The DASH Outcome Measure User's Manual Toronto, Ontario: Institute for Work \& Health 2002:5.

21. Streiner DL and Norman GR: Health measurement scales: A practical guide to their development and use Oxford: Oxford University Press 1995:64.

22. Nunnally JC and Bernstein IH: Psychometric theory New York: McGraw-Hill 1994:265.

\section{Pre-publication history}

The pre-publication history for this paper can be accessed here:

http://www.biomedcentral.com/1471-2474/4/11/prepub

Publish with Bio Med Central and every scientist can read your work free of charge

"BioMed Central will be the most significant development for disseminating the results of biomedical research in our lifetime. "

Sir Paul Nurse, Cancer Research UK

Your research papers will be:

- available free of charge to the entire biomedical community

- peer reviewed and published immediately upon acceptance

- cited in PubMed and archived on PubMed Central

- yours - you keep the copyright 